\title{
Model-Driven Information Flow Security for Component-Based Systems ${ }^{\star}$
}

\author{
Najah Ben Said ${ }^{1}$, Takoua Abdellatif ${ }^{2}$, Saddek Bensalem $^{1}$, and Marius Bozga ${ }^{1}$ \\ 1 UJF-Grenoble 1/CNRS, VERIMAG UMR 5104, Grenoble, 38041, France \\ 2 Sousse University, ESSTHS, Hammam Sousse, Tunisia
}

\begin{abstract}
This paper proposes a formal framework for studying information flow security in component-based systems. The security policy is defined and verified from the early steps of the system design. Two kinds of non-interference properties are formally introduced and for both of them, sufficient conditions that ensures and simplifies the automated verification are proposed. The verification is compositional, first locally, by checking the behavior of every atomic component and then globally, by checking the inter-components communication and coordination. The potential benefits are illustrated on a concrete case study about constructing secure heterogeneous distributed systems.
\end{abstract}

Keywords: component-based systems, information flow security, noninterference, unwinding conditions, automated verification.

\section{Introduction}

The amount and complexity of nowadays conceived systems and software knows a continuous increase. Information protection and secure information flow between these systems is paramount and represent a great design challenge. Model driven security (MDS) BDL06 is an innovative approach that tend to solve system-level security issues by providing an advanced modeling process representing security requirements at a high level of abstraction. Indeed, MDS guarantees separation of concerns between functional and security requirements, from early phases of the system development till final implementation.

Information flow security can be ensured using various mechanisms. Amongst the first approaches considered, ones find access control policies SSM98Kuh98, that allow protecting data confidentiality by limiting access to data to be read or modified only by authorized users. Unfortunately, these mechanisms have been proven incomplete and limited since only by preventing the direct access to data, indirect (implicit) information flows are still possible given rise to the so called covert channels SQSL05. As an alternative, non-interference has been studied as a global property to characterize and to develop techniques ensuring

\footnotetext{
* The research leading to these results has received funding from the European Community's Seventh Framework Programme [FP7/2007-2013] under grant agreement ICT-318772 (D-MILS).
}

S. Bensalem et al. (Eds.): FPS 2014 (Sifakis Festschrift), LNCS 8415, pp. 1-20, 2014.

(C) Springer-Verlag Berlin Heidelberg 2014 
information flow security. Initially defined by Goguen and Meseguer GM82, non-interference ensures that the system's secret information does not affect its public behavior.

In this work, we adapt the MDS approach to develop a component-based framework, named secBIP, that guarantees automated verification and implementation of secure information flow systems with respect to specific definition of non-interference. In general, component-based frameworks allow the construction of complex systems by composition of atomic components with communication and coordination operators. That is, systems are obtained from unitary atomic components that can be independently deployed and composed with other components. Component-based frameworks are usually well adopted for managing key issues for functional design including heterogeneity of components, distribution aspects, performance issues, etc. Nonetheless, the use of componentbased frameworks is also beneficial for establishing information flow security. Particularly, the explicit system architecture allows tracking easily intra and inter-components information flow.

The secBIP framework is built as an extension of the $B I P$ BBS06 $\mathrm{BBB}^{+} 11$ framework encompassing information flow security. secBIP allows to create systems that are secure by construction if certain local conditions hold for composed components. The secBIP extension includes specific annotations for classification of both data and interactions. Thanks to the explicit use of composition operators in BIP, the information flow is easily tracked within models and security requirements can be established in a compositional manner, first locally, by checking the behavior of atomic components and then globally, by checking the communication and coordination inter-components.

Information flow security has been traditionally studied separately for language-based models [SS01 SV98] (see also the survey SM03) and trace-based models McC88 McL94ZL97Man00]. While the former mostly focus on verification of data-flow security properties in programming languages, the latter is treating security in event-based systems. In $\sec B I P$, we achieve a useful combination between both aspects, data-flow and event-flow security, in a single semantics model. We introduce and distinguish two types of non-interference, respectively event non-interference and data non-interference. For events, non-interference states that the observation of public events should not allow to deduce any information about the occurrence of secret events. For data, it states that there is no leakage of secret data into public ones.

The paper is structured as follows. Section 2 recalls the main concepts of the component-based framework adopted in this work. In section 3. we formally introduce the security extension and we provide the two associated definitions of non-interference, respectively for data flows and event flows. Next, in section 4 we formally establish non-interference based on unwinding relations and we provide sufficient conditions that facilitate its automatic verification. In section 5. we provide a use-case as illustrative example. Section 6 discusses the related work and section 7 concludes and presents some lines for future work. All the proofs of technical results are given in the appendix. 


\section{Component-Based Design}

The secBIP framework is built as an extension of the $B I P$ framework introduced in BBS06. BIP stands for Behavior, Interaction and Priority, that is, the three layers used for the definition of components and their composition in this framework. BIP allows the construction of complex, hierarchically structured models from atomic components characterized by their behavior and their interfaces. Such components are transition systems enriched with data. Transitions are used to move from a source to a destination location. Each time a transition is taken, component data (variables) may be assigned new values, computed by user-defined functions (in C). Atomic components are composed by layered application of interactions and priorities. Interactions express synchronization constraints and do the transfer of data between the interacting components. Priorities are used to filter amongst possible interactions and to steer system evolution so as to meet performance requirements e.g., to express scheduling policies.

In this section, we briefly recall the key concepts of $B I P$ which are further relevant for dealing with information flow security. In particular, we give a formal definition of atomic components and their composition through multiparty interactions. Priorities are not considered in this work.

\subsection{Atomic Components}

Definition 1 (atomic component). An atomic component $B$ is a tuple $(L$, $X, P, T)$ where $L$ is a set of locations, $X$ is a set of variables, $P$ is a set of ports and $T \subseteq L \times P \times L$ is a set of port labelled transitions. For every port $p \in P$, we denote by $X_{p}$ the subset of variables exported and available for interaction through $p$. For every transition $\tau \in T$, we denote by $g_{\tau}$ its guard, that is, a boolean expression defined on $X$ and by $f_{\tau}$ its update function, that is, a parallel assignment $\left\{x:=e_{\tau}^{x}\right\}_{x \in X}$ to variables of $X$.

Figure 1 provides an example of an atomic component. It contains two control locations $l_{1}$ and $l_{2}$ and two ports $p_{1}$ and $p_{2}$. The transition labeled with $p_{1}$ can take place only if the guard $(0<x)$ is true. When the transition takes place, the variable $y$ is recalculated as some function of $x$.

Let $\mathcal{D}$ be the data domain of variables. Given a set of variables $Y$, we call valuation on $Y$ any function $\mathbf{y}: Y \rightarrow \mathcal{D}$ mapping variables to data. We denote by $\mathbf{Y}$ the set of all Fig. 1. Atomic Component in BIP valuations defined on $Y$.

Definition 2 (atomic component semantics). The semantics of an atomic component $B=(L, X, P, T)$ is defined as the labelled transition system $\operatorname{LTS}(B)=$ 
$\left(Q_{B}, \Sigma_{B}, \underset{B}{\rightarrow}\right)$ where the set of states $Q_{B}=L \times \mathbf{X}$, the set of labels is $\Sigma_{B}=P \times \mathbf{X}$ and the set of labelled transitions $\underset{B}{\rightarrow}$ is defined by the rule:

$$
\text { Атом } \frac{\tau=\ell \stackrel{p}{\rightarrow} \ell^{\prime} \in T \quad \mathbf{x}_{p}^{\prime \prime} \in \mathbf{X}_{p} \quad g_{\tau}(\mathbf{x}) \quad \mathbf{x}^{\prime}=f_{\tau}\left(\mathbf{x}\left[X_{p} \leftarrow \mathbf{x}_{p}^{\prime \prime}\right]\right)}{(\ell, \mathbf{x}) \frac{p\left(\mathbf{x}_{p}^{\prime \prime}\right)}{B}\left(\ell^{\prime}, \mathbf{x}^{\prime}\right)}
$$

That is, $\left(\ell^{\prime}, \mathbf{x}^{\prime}\right)$ is a successor of $(\ell, \mathbf{x})$ labelled by $p\left(\mathbf{x}_{p}^{\prime \prime}\right)$ iff $(1) \tau=\ell \stackrel{p}{\rightarrow} \ell^{\prime}$ is a transition of $T,(2)$ the guard $g_{\tau}$ holds on the current valuation $\mathbf{x},(3) \mathbf{x}_{p}^{\prime \prime}$ is a valuation of exported variables $X_{p}$ and (4) $\mathbf{x}^{\prime}=f_{\tau}\left(\mathbf{x}\left[X_{p} \leftarrow \mathbf{x}_{p}^{\prime \prime}\right]\right)$ meaning that, the new valuation $\mathbf{x}^{\prime}$ is obtained by applying $f_{\tau}$ on $\mathbf{x}$ previously modified according to $\mathbf{x}_{p}^{\prime \prime}$. Whenever a $p$-labelled successor exist in a state, we say that $p$ is enabled in that state.

\subsection{Composite Components}

Composite components are obtained by composing an existing set of atomic components $\left\{B_{i}=\left(L_{i}, X_{i}, P_{i}, T_{i}\right)\right\}_{i=1, n}$ trough specific composition operators. We consider that atomic components have pairwise disjoint sets of states, ports, and variables i.e., for any two $i \neq j$ from $\{1 . . n\}$, we have $L_{i} \cap L_{j}=\emptyset, P_{i} \cap P_{j}=\emptyset$, and $X_{i} \cap X_{j}=\emptyset$. We denote $P=\bigcup_{i=1}^{n} P_{i}$ the set of all the ports, $L=\bigcup_{i=1}^{n} L_{i}$ the set of all locations, and $X=\bigcup_{i=1}^{n} X_{i}$ the set of all variables.

Definition 3 (interaction). An interaction a between atomic components is a triple $\left(P_{a}, G_{a}, F_{a}\right)$, where $P_{a} \subseteq P$ is a set of ports, $G_{a}$ is a guard, and $F_{a}$ is an update function. By definition, $P_{a}$ uses at most one port of every component, that is, $\left|P_{i} \cap P_{a}\right| \leq 1$ for all $i \in\{1 . . n\}$. Therefore, we simply denote $P_{a}=\left\{p_{i}\right\}_{i \in I}$, where $I \subseteq\{1 . . n\}$ contains the indices of the components involved in a and for all $i \in I, p_{i} \in P_{i} . G_{a}$ and $F_{a}$ are both defined on the variables exported by the ports in $P_{a}$ (i.e., $\bigcup_{p \in P_{a}} X_{p}$ ).

Definition 4 (composite component). A composite component $C=\gamma\left(B_{1}\right.$, $\left.\ldots, B_{n}\right)$ is obtained by applying a set of interactions $\gamma$ to a set of atomic components $B_{1}, \ldots B_{n}$.

Figure 2 presents a classical Producer-Buffer-Consumer example modeled in BIP. It consists of three atomic components, namely Producer, Buffer and Consumer. The Buffer is a shared memory placeholder, which is accessible by both the Producer and the Consumer. It holds into the local variable $x$ the number of items available. The Buffer interacts with the Producer (res. Consumer) on the put (resp. get) interaction. On the put interaction, an item is added to the Buffer and $x$ is incremented. On the get interaction, the Consumer removes an item from the Buffer, if at least one exists (the guard $[x \geq 1]$ ), and $x$ is decremented. Finally, the transitions labeled produce and consume do not require synchronization - they are executed alone (on singleton port interactions) by the respective components. 


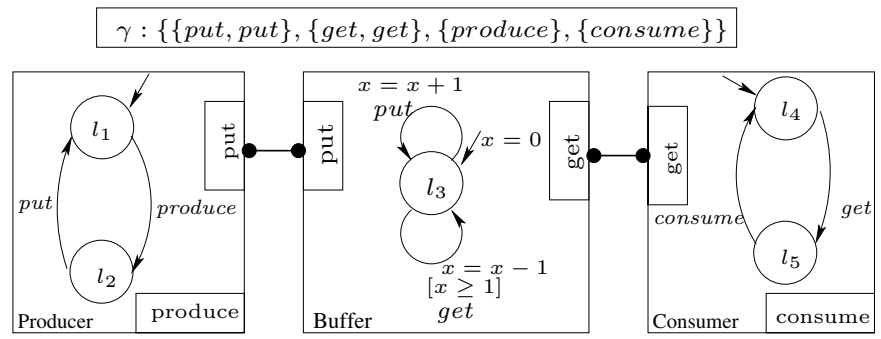

Fig. 2. BIP model of the Producer-Buffer-Consumer example

Definition 5 (composite component semantics). Let $C=\gamma\left(B_{1}, \ldots, B_{n}\right)$ be a composite component. Let $B_{i}=\left(L_{i}, X_{i}, P_{i}, T_{i}\right)$ and $\operatorname{LTs}\left(B_{i}\right)=\left(Q_{i}, \Sigma_{i}, \underset{B_{i}}{\longrightarrow}\right)$ their semantics, for all $i=1, n$. The semantics of $C$ is the labelled transition system $\operatorname{LTS}(C)=\left(Q_{C}, \Sigma_{C}, \underset{C}{\longrightarrow}\right)$ where the set of states $Q_{C}=\otimes_{i=1}^{n} Q_{i}$, the set of labels $\Sigma_{C}=\gamma$ and the set of labelled transitions $\underset{C}{\rightarrow}$ is defined by the rule:

$a=\left(\left\{p_{i}\right\}_{i \in I}, G_{a}, F_{a}\right) \in \gamma \quad G_{a}\left(\left\{\mathbf{x}_{p_{i}}\right\}_{i \in I}\right) \quad\left\{\mathbf{x}_{p_{i}}^{\prime \prime}\right\}_{i \in I}=F_{a}\left(\left\{\mathbf{x}_{p_{i}}\right\}_{i \in I}\right)$
$\forall i \in I .\left(\ell_{i}, \mathbf{x}_{i}\right) \frac{p_{i}\left(\mathbf{x}_{p_{i}}^{\prime \prime}\right)}{B_{i}}\left(\ell_{i}^{\prime}, \mathbf{x}_{i}^{\prime}\right) \quad \forall i \notin I .\left(\ell_{i}, \mathbf{x}_{i}\right)=\left(\ell_{i}^{\prime}, \mathbf{x}_{i}^{\prime}\right)$
$\operatorname{Comp~} \frac{\left.\forall\left(\ell_{1}, \mathbf{x}_{1}\right), \ldots,\left(\ell_{n}, \mathbf{x}_{n}\right)\right) \underset{C}{\vec{a}}\left(\left(\ell_{1}^{\prime}, \mathbf{x}_{1}^{\prime}\right), \ldots,\left(\ell_{n}^{\prime}, \mathbf{x}_{n}^{\prime}\right)\right)}{}$

For each $i \in I, \mathbf{x}_{p_{i}}$ above denotes the valuation $\mathbf{x}_{i}$ restricted to variables of $X_{p_{i}}$.

The rule expresses that a composite component $C=\gamma\left(B_{1}, \ldots, B_{n}\right)$ can execute an interaction $a \in \gamma$ enabled in state $\left(\left(\ell_{1}, \mathbf{x}_{1}\right), \ldots,\left(\ell_{n}, \mathbf{x}_{n}\right)\right)$, iff (1) for each $p_{i} \in P_{a}$, the corresponding atomic component $B_{i}$ can execute a transition labelled by $p_{i}$, and (2) the guard $G_{a}$ of the interaction holds on the current valuation of variables exported on ports participating in $a$. Execution of interaction $a$ triggers first the update function $F_{a}$ which modifies variables exported by ports $p_{i} \in P_{a}$. The new values obtained, encoded in the valuation $\mathbf{x}_{p_{i}}^{\prime \prime}$, are then used by the components' transitions. The states of components that do not participate in the interaction remain unchanged.

Any finite sequences of interactions $w=a_{1} \ldots a_{k} \in \gamma^{*}$ executable by the composite component starting at some given initial state $q_{0}$ is named a trace. The set of all traces $w$ from state $q_{0}$ is denoted by $\operatorname{TRACES}\left(C, q_{0}\right)$.

\section{Information Flow Security}

We explore information flow policies DD77 BLP76 GM82] with focus on the noninterference property. In order to track information we adopt the classification technique and we define a classification policy where we annotate the information by assigning security levels to different parts of secBIP model (data variables, 
ports and interactions). The policy describes how information can flow from one classification with respect to the other.

As an example, we can classify public information as a Low $(L)$ security level and secret (confidential) information as High $(H)$ security level. Intuitively High security level is more restrictive than Low security level and we denote it by $L \subseteq H$. In general, security levels are elements of a security domain, defined as follows:

Definition 6 (security domain). A security domain is a lattice of the form $\langle S, \subseteq, \cup, \cap\rangle$ where:

- $S$ is a finite set of security levels.

$-\subseteq$ is a partial order "can flow to" on $S$ that indicates that information can flow from one security level to an equal or a more restrictive one.

$-\cup$ is a "join" operator for any two levels in $S$ and that represents the upper bound of them.

$-\cap$ is a "meet" operator for any two levels in $S$ and that represents the lower bound of them.

As an example, consider the set $S=$ $\left\{L, M_{1}, M_{2}, H\right\}$ of security levels that are governed by the "can flow to" relation $L \subseteq$ $M_{1}, L \subseteq M_{2}, M_{1} \subseteq H$ and $M_{2} \subseteq H . M_{1}$ and $M_{2}$ are incomparable and we note $M_{1} \nsubseteq M_{2}$ and $M_{1} \nsupseteq M_{2}$. This security domain is graphically illustrated in Figure 3 .

Let $C=\gamma\left(B_{1}, \ldots B_{n}\right)$ be a composite component, fixed. Let $X$ (resp. $P$ ) be the set of all variables (resp. ports) defined in all atomic components $\left(B_{i}\right)_{i=1, n}$.

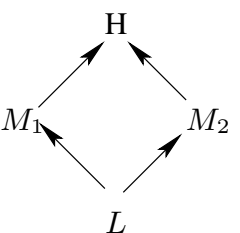

Fig. 3. Example of security domain

Let $\langle S, \subseteq, \cup, \cap\rangle$ be a security domain, fixed.

Definition 7 (security assignment). A security assignment for component $C$ is a mapping $\sigma: X \cup P \cup \gamma \rightarrow S$ that associates security levels to variables, ports and interactions such that, moreover, the security levels of ports matches the security levels of interactions, that is, for all $a \in \gamma$ and for all $p \in P$ it holds $\sigma(p)=\sigma(a)$.

In atomic components, the security levels considered for ports and variables allow to track intra-component information flows and control the intermediate computation steps. Moreover, inter-components communication, that is, interactions with data exchange, are tracked by the security levels assigned to interactions.

In order to formally introduce the two notions of non-interference for secBIP models we need few additional notations, as follows. Let $\sigma$ be a security assignment for $C$, fixed. 
For a security level $s \in S$, we define $\gamma \downarrow_{s}^{\sigma}$ the restriction of $\gamma$ to interactions with security level at most $s$ that is formally, $\gamma \downarrow_{s}^{\sigma}=\{a \in \gamma \mid \sigma(a) \subseteq s\}$.

For a security level $s \in S$, we define $\left.w\right|_{s} ^{\sigma}$ the projection of a trace $w \in \gamma^{*}$ to interactions with security level lower or equal to $s$. Formally, the projection is recursively defined on traces as $\left.\epsilon\right|_{s} ^{\sigma}=\epsilon,\left.(a w)\right|_{s} ^{\sigma}=a\left(\left.w\right|_{s} ^{\sigma}\right)$ if $\sigma(a) \subseteq s$ and $\left.(a w)\right|_{s} ^{\sigma}=\left.w\right|_{s} ^{\sigma}$ if $\sigma(a) \nsubseteq s$. The projection operator $\left.\right|_{s} ^{\sigma}$ is naturally lifted to sets of traces $W$ by taking $\left.W\right|_{s} ^{\sigma}=\left\{\left.w\right|_{s} ^{\sigma} \mid w \in W\right\}$.

For a security level $s \in S$, we define the equivalence $\approx_{s}^{\sigma}$ on states of $C$. Two states $q_{1}, q_{2}$ are equivalent, denoted by $q_{1} \approx_{s}^{\sigma} q_{2}$ iff (1) they coincide on variables having security levels at most $s$ and (2) they coincide on control locations having outgoing transitions labeled with ports with security level at most $s$.

We are now ready to define the two notions of non-interference.

Definition 8 (event non-interference). The security assignment $\sigma$ ensures event non-interference of $\gamma\left(B_{1}, \ldots, B_{n}\right)$ at security level $s$ iff,

$$
\forall q_{0} \in Q_{C}^{0}:\left.\operatorname{TRACES}\left(\gamma\left(B_{1}, \ldots, B_{n}\right), q_{0}\right)\right|_{s} ^{\sigma}=\operatorname{TRACES}\left(\left(\gamma \downarrow_{s}^{\sigma}\right)\left(B_{1}, \ldots, B_{n}\right), q_{0}\right)
$$

Event non-interference ensures isolation/security at interaction level. The definition excludes the possibility to gain any relevant information about the occurrences of interactions (events) with strictly greater (or incomparable) levels than $s$, from the exclusive observation of occurrences of interactions with levels lower or equal to $s$. That is, an external observer is not able to distinguish between the case where such higher interactions are not observable on execution traces and the case these interactions have been actually statically removed from the composition. This definition is very close to Rushby's Rus92 definition for transitive non-interference. But, let us remark that event non-interference is not concerned about the protection of data.

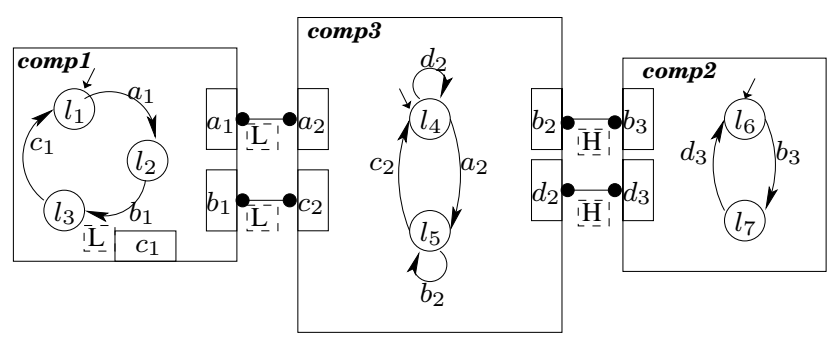

Fig. 4. Example for event non-interference

Example 1. Figure 4 presents a simple illustrative example for event noninterference. The model consists of three atomic components $\operatorname{comp}_{i, i=1,2,3}$. Different security levels have been assigned to ports and interactions: $c o m p_{1}$ is a low security component, $c o m p_{2}$ is a high security component, and $c o m p_{3}$ is mixed security component. The security levels are represented by dashed squares related to interactions, internal ports and variables. As a convention, we apply high $(H)$ 
level for secret data and interactions and low $(L)$ level for public ones. The set of traces is represented by the automaton in Figure 5 (a). The set of projected execution traces at security level $L$ is represented by the automaton depicted in Figure 5 (b). This set is equal to the set of traces obtained by restricted composition, that is, using interaction with security level at most $L$ and depicted in Figure 5 (c). Therefore, this example satisfies the event non-interference condition at level $L$.

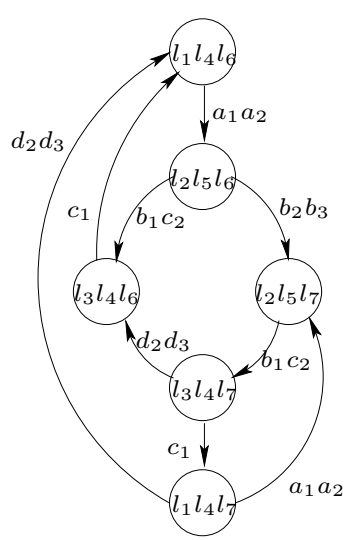

(a)

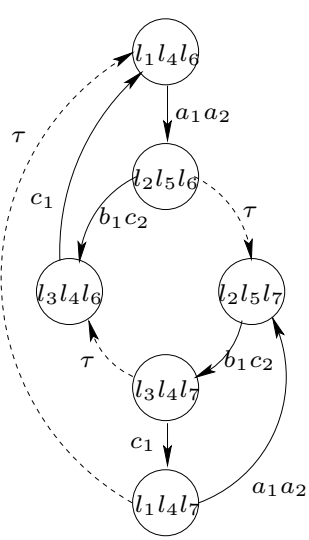

(b)

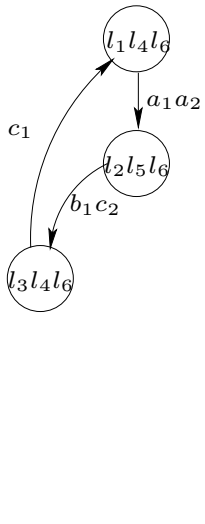

(c)

Fig. 5. Sets of traces represented as automata

Definition 9 (data non-interference). The security assignment $\sigma$ ensures data non-interference of $C=\gamma\left(B_{1}, \ldots, B_{n}\right)$ at security level $s$ iff,

$$
\begin{aligned}
& \forall q_{1}, q_{2} \in Q_{C}^{0}: q_{1} \approx_{s}^{\sigma} q_{2} \Rightarrow \\
& \forall w_{1} \in \operatorname{TRACES}\left(C, q_{1}\right), w_{2} \in \operatorname{TRACES}\left(C, q_{2}\right):\left.w_{1}\right|_{s} ^{\sigma}=\left.w_{2}\right|_{s} ^{\sigma} \Rightarrow \\
& \forall q_{1}^{\prime}, q_{2}^{\prime} \in Q_{C}: q_{1} \frac{w_{1}}{C} q_{1}^{\prime} \wedge q_{2} \frac{w_{2}}{C} q_{2}^{\prime} \Rightarrow q_{1}^{\prime} \approx_{s}^{\sigma} q_{2}^{\prime}
\end{aligned}
$$

Data non-interference provides isolation/security at data level. The definition ensures that, all states reached from initially indistinguishable states at security level $s$, by execution of arbitrary but identical traces whenever projected at level $s$, are also indistinguishable at level $s$. That means that observation of all variables and interactions with level $s$ or lower excludes any gain of relevant information about variables at higher (or incomparable) level than $s$. Compared to event non-interference, data non-interference is a stronger property that considers the system's global states (local states and valuation of variables) and focus on their equivalence along identical execution traces (at some security level).

Example 2. Figure 6 presents an extension with data variables of the previous example from Figure 4. We consider the following two traces $w_{1}=\left\langle a_{1} a_{2}, b_{2} b_{3}\right.$, $\left.c_{2} b_{1}, d_{2} d_{3}, c_{1}, a_{2} a_{1}\right\rangle$ and $w_{2}=\left\langle a_{1} a_{2}, b_{2} b_{3}, c_{2} b_{1}, c_{1}, a_{2} a_{1}\right\rangle$ that start from the initial 
state $\left(\left(l_{1}, u=0, v=0\right),\left(l_{4}, x=0, y=0\right),\left(l_{6}, z=0, w=0\right)\right)$. Although the projected traces at level $L$ are equal, that is, $\left.w_{1}\right|_{L} ^{\sigma}=\left.w_{2}\right|_{L} ^{\sigma}=\left\langle a_{1} a_{2}, c_{2} b_{1}, c_{1}, a_{1} a_{2}\right\rangle$, the reached states by $w_{1}$ and $w_{2}$ are different, respectively $\left(\left(l_{2}, u=4, v=\right.\right.$ $\left.2),\left(l_{5}, x=3, y=2\right),\left(l_{6}, z=1, w=1\right)\right)$ and $\left(\left(l_{2}, u=4, v=2\right),\left(l_{5}, x=2, y=\right.\right.$ $\left.2),\left(l_{7}, z=1, w=0\right)\right)$ and moreover non-equivalent at low level $L$. Hence, this example is not data non-interferent at level $L$.

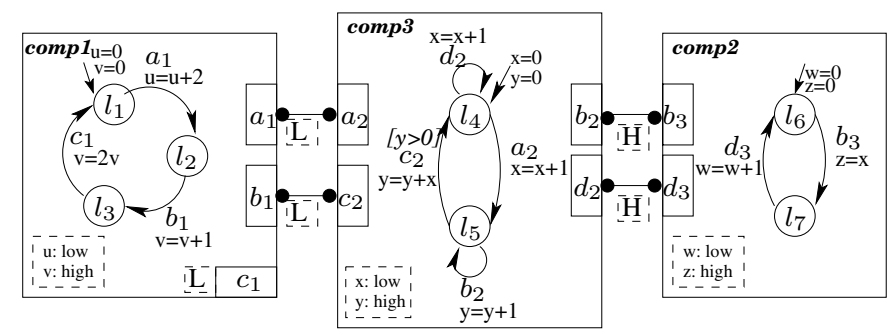

Fig. 6. Example for data non-interference

Definition 10 (secure component). A security assignment $\sigma$ is secure for a component $\gamma\left(B_{1}, \ldots, B_{n}\right)$ iff it ensures both event and data non-interference, at all security levels $s \in S$.

\section{Automated Verification of Non-interference}

We propose hereafter an automated verification technique of non-interference for secBIP models based on the so-called unwinding conditions. These conditions were first introduced by Goguen and Meseguer for the verification of transitive non-interference for deterministic systems GM82. In general, the unwinding approach reduces the verification of information flow security to the existence of certain unwinding relation. This relation is usually an equivalence relation on system states that respects some additional properties on atomic execution steps, which are shown sufficient to imply non-interference. In the case of $\sec B I P$, the additional properties are formulated in terms of individual interactions/events and therefore easier to handle.

Let $C=\gamma\left(B_{1}, \ldots, B_{n}\right)$ be a composite component and let $\sigma$ be a security assignment for $C$.

Definition 11 (unwinding relation). An equivalence $\sim_{s}$ on states of $C$ is called an unwinding relation for $\sigma$ at security level $s$ iff the two following conditions hold:

1. local consistency

$\forall q, q^{\prime} \in Q_{C}: \forall a \in \gamma: q \underset{C}{\stackrel{a}{\longrightarrow}} q^{\prime} \Rightarrow \sigma(a) \subseteq s \vee q \sim_{s} q^{\prime}$ 
2. output and step consistency

$$
\begin{aligned}
& \forall q_{1}, q_{2}, q_{1}^{\prime} \in Q_{C}: \forall a \in \gamma: \\
& q_{1} \sim_{s} q_{2} \wedge q_{1} \underset{C}{\vec{C}} q_{1}^{\prime} \wedge \sigma(a) \subseteq s \Rightarrow \\
& \quad \exists q_{2}^{\prime} \in Q_{C}: q_{2} \underset{\vec{C}}{\stackrel{a}{\longrightarrow}} q_{2}^{\prime} \wedge \\
& \forall q_{2}^{\prime} \in Q_{C}: q_{2} \underset{C}{\stackrel{a}{\longrightarrow}} q_{2}^{\prime} \Rightarrow q_{1}^{\prime} \sim_{s} q_{2}^{\prime}
\end{aligned}
$$

The existence of unwinding relations is tightly related to non-interference. The following two theorems formalize this relation for the two types of noninterference defined. Let $C$ be a composite component and $\sigma$ a security assignment.

Theorem 1 (event non-interference). If an unwinding relation $\sim_{s}$ exists for the security assignment $\sigma$ at security level $s$, then $\sigma$ ensures event noninterference of $C$ at level $s$.

Theorem 2 (data non-interference). If the equivalence relation $\approx_{s}^{\sigma}$ is also an unwinding relation for the security assignment $\sigma$ at security level $s$, then $\sigma$ ensures data non-interference of $C$ at level s.

The two theorems above are used to derive a practical verification method of non-interference using unwinding. We provide hereafter sufficient syntactic conditions ensuring that indeed the unwinding relations $\sim_{s}$ and $\approx_{s}$ exist on the system states. These conditions aim to effectively reduce the verification of non-interference to the checking on local constraints on both transitions (intra-component conditions) and interactions (inter-component conditions). Especially, they give an direct way to automate the verification.

Definition 12 (security conditions). Let $C=\gamma\left(B_{1}, \ldots, B_{n}\right)$ be a composite component and let $\sigma$ be a security assignment. We say that $C$ satisfies the security conditions for security assignment $\sigma$ iff:

(i) the security assignment of ports, in every atomic component $B_{i}$ is locally consistent, that is:

- for every pair of causal transitions:

$$
\begin{gathered}
\forall \tau_{1}, \tau_{2} \in T_{i}: \tau_{1}=\ell_{1} \stackrel{p_{1}}{\longrightarrow} \ell_{2}, \tau_{2}=\ell_{2} \stackrel{p_{2}}{\longrightarrow} \ell_{3} \quad \Rightarrow \\
\ell_{1} \neq \ell_{2} \Rightarrow \sigma\left(p_{1}\right) \subseteq \sigma\left(p_{2}\right)
\end{gathered}
$$

- for every pair of conflicting transitions:

$$
\begin{gathered}
\forall \tau_{1}, \tau_{2} \in T_{i}: \tau_{1}=\ell_{1} \stackrel{p_{1}}{\longrightarrow} \ell_{2}, \tau_{2}=\ell_{1} \stackrel{p_{2}}{\longrightarrow} \ell_{3} \quad \Rightarrow \\
\ell_{1} \neq \ell_{2} \Rightarrow \sigma\left(p_{1}\right) \subseteq \sigma\left(p_{2}\right)
\end{gathered}
$$

(ii) all assignments $x:=e$ occurring in transitions within atomic components and interactions are sequential consistent, in the classical sense:

$$
\forall y \in u s e(e): \sigma(y) \subseteq \sigma(x)
$$


(iii) variables are consistently used and assigned in transitions and interactions, that is,

$$
\begin{gathered}
\forall \tau \in \cup_{i=1}^{n} T_{i} \quad \forall x, y \in X: x \in \operatorname{def}\left(f_{\tau}\right), y \in \operatorname{use}\left(g_{\tau}\right) \quad \Rightarrow \\
\sigma(y) \subseteq \sigma\left(p_{\tau}\right) \subseteq \sigma(x) \\
\forall a \in \gamma \quad \forall x, y \in X: x \in \operatorname{def}\left(F_{a}\right), y \in \operatorname{use}\left(G_{a}\right) \quad \Rightarrow \\
\sigma(y) \subseteq \sigma(a) \subseteq \sigma(x)
\end{gathered}
$$

(iv) all atomic components $B_{i}$ are port deterministic:

$$
\begin{aligned}
& \forall \tau_{1}, \tau_{2} \in T_{i}: \tau_{1}=\ell_{1} \stackrel{p}{\rightarrow} \ell_{2}, \tau_{2}=\ell_{1} \stackrel{p}{\rightarrow} \ell_{3} \Rightarrow \\
& \quad\left(g_{\tau_{1}} \wedge g_{\tau_{2}}\right) \text { is unsatisfiable }
\end{aligned}
$$

The first family of conditions (i) is similar to Accorsi's conditions AL12 for excluding causal and conflicting places for Petri net transitions having different security levels. Similar conditions have been considered in [FG01FGF09] and lead to more specific definitions of non-interferences and bisimulations on annotated Petri nets. The second condition (ii) represents the classical condition needed to avoid information leakage in sequential assignments. The third condition (iii) tackles covert channels issues. Indeed, (iii) enforces the security levels of the data flows which have to be consistent with security levels of the ports or interactions (e.g., no low level data has to be updated on a high level port or interaction). Such that, observations of public data would not reveal any secret information. Finally, conditions $(i v)$ enforces deterministic behavior on atomic components.

The relation between the syntactic security conditions and the unwinding relations is precisely captured by the following theorem.

Theorem 3 (unwinding theorem). Whenever the security conditions hold, the equivalence relation $\approx_{s}^{\sigma}$ is an unwinding relation for the security assignment $\sigma$, at all security level $s$.

The following result is the immediate consequence of theorems 1, 2 and 3 .

Corollary 1. Whenever the security conditions hold, the security assignment $\sigma$ is secure for the component $C$.

\section{Case Study: Web Service Reservation System}

We illustrate the $\sec B I P$ framework to handle information flow security issues for a classical example, the web service reservation system proposed in HV06. A businessman, living in France, plans to go to Berlin for a private and secret mission. To organize his travel, he uses an intelligent web service who contacts two travel agencies: The first agency, AgencyA, arranges flights in Europe and the second agency, AgencyB, arranges flights exclusively to Germany. The reservation service obtains in return specific flight information and their corresponding prices and chooses the flight that is more convenient for him. 
In this example, there are two types of interference that can occur, (1) datainterference since learning the flight price may reveal the flight destination and (2) event interference, since observing the interaction with AgencyB can reveal the destination as well. Thus, to keep the mission private, the flight prices and interactions with $\operatorname{AgencyB}$ have to be kept confidential.

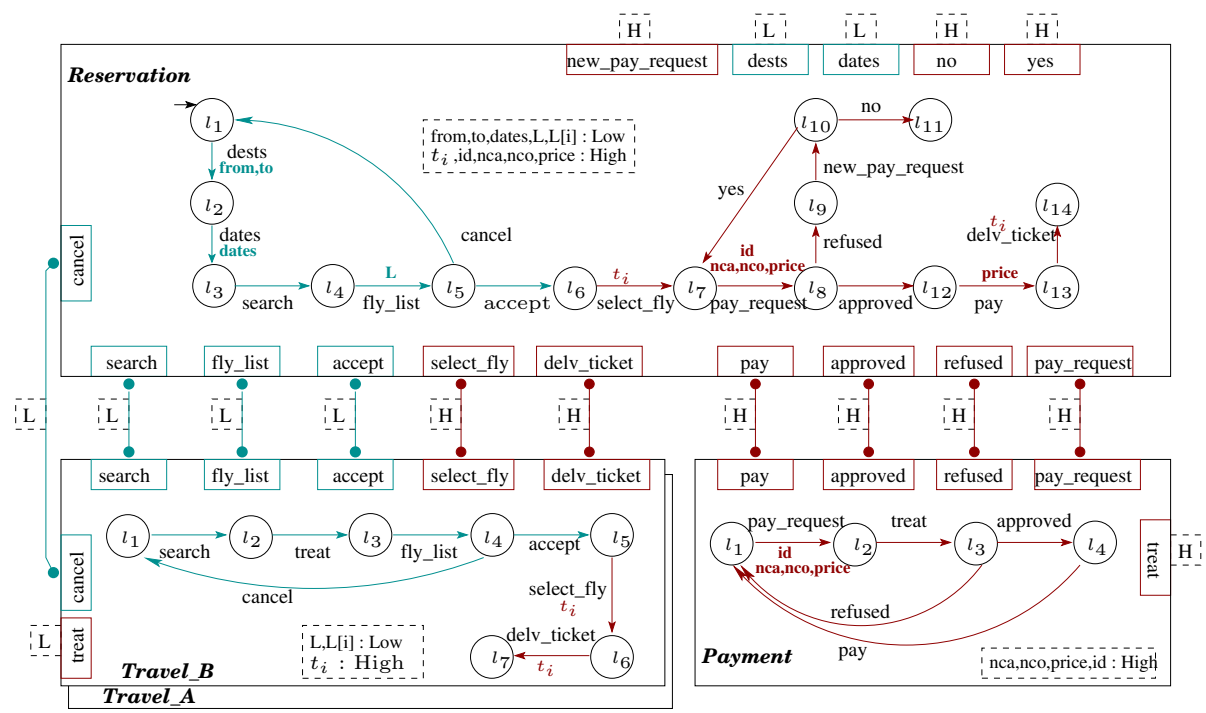

Fig. 7. Model of Reservation Web Service in $\sec B I P$

The modeling of the system using secBIP involves two main distinct steps: first, functional requirements modeling reflecting the system behavior, and second, security annotations enforcing the desired security policy. The model of the system has four components denoted: Travel_A and Travel_B who are instances from the same component and correspond respectively to AgencyA and AgencyB, and components Reservation and Payment. To avoid Figure 7 cluttering, we did not represent the interactions with Travel_A component. Search parameters are supplied by a user through the Reservation component ports dests and dates to which we associate respectively variables (from, to) and dates. Next, through search interaction, Reservation component contacts Travel_B component to search for available flights and obtains in return a list $L$ of specific flights with their corresponding prices. Thereafter, Reservation component selects a ticket $t_{i}$ from the list $L$ and requests the Payment component to perform the payment.

All the search parameters from, to, dates, as well as the flights list $L$ are set to low since users are not identified while sending these queries. Other sensitive data like the selected flight $t_{i}$, the price variable $p$ and the payment parameters (identity $i d$, credit card variable cna and code number cno) are set to high. Internal ports dests and dates as well as search, fly_list, accept interactions are set to low since these interactions (events) do not reveal any information about the 
client private trip. However, the select_fly interaction must be set to high since the observation of the selection event from AgencyB allow to deduce deduce the client destination. In the case of a selected flight from AgencyA, the select_fly interaction could be set to low since, in this case, the destination could not be deduced just from the event occurrence.

We recall that any system can be proven non-interferent iff it satisfies the syntactic security conditions from Definition 12, Indeed, these conditions hold for the system model depicted in Figure 7 In particular, it can be easily checked that all assignments occurring in transitions within atomic component as well as within interactions are sequential consistent. For example, at the select_fly interaction we assign a low level security item from the flight list $L$ to a high security level variable $t i$, formally $t_{i}=L[i]$. Besides, the security levels assignments to ports exclude inconsistencies due to causal and conflicting transitions, in all atomic components.

\section{Related Work}

Non-interference properties have been already studied using different modelbased approaches. Recently, SS12] adapted an MDS method for handling information flow security using UML sequence diagrams. Additionally, Petri-nets have been extensively used for system modeling and information flow security verifications tools such as InDico [AWD11] have been developed. A componentbased model has been proposed in [ASRL11] and used to study implementation issues of secure information flows. Our presented work on $\operatorname{secBIP}$ is however different and original in several respects.

First, secBIP is a formal framework. Unlike UML, system's runtime behavior is always meaningfully defined and can be formally analyzed. Moreover, secBIP provides a system construction methodology for complex systems. Indeed, big systems are functionally decomposed into multiple sub-components communicating through well-defined interactions. Such a structural decomposition of the system is usually not available on Petri-nets models.

Second, secBIP handles both event and data-flow non-interference, in a single semantic model. To the best of our knowledge, these properties have never been jointly considered for component-based models. Nevertheless, the need to consider together event and data flow non-interference has been recently identified in the existing literature. The bottom line is that preserving the safety of data flow in a system does not necessarily preserve safe observability on system's public behavior (i.e., secret/private executions may have an observable impact on system public events). The issue has been recently considered in [AL12, for data leaks and information leaks in business processes based on system's data-flows and work-flows. Also, BBMP08 showed that formal verification of the system's event behavior is not sufficient to guarantee specific data properties. Furthermore, FRS05] attempted to fill the gap between respectively language-based and process calculusbased information security and make an explicit distinction between preventing the data leakage through the execution of programs and preventing secret events from being revealed in inter-process communications. 
Third, compared to security-typed programming languages jif ZZNM02 and operating systems $\left.\mathrm{KYB}^{+} 07 \mathrm{ZBWM} 08 \mathrm{EKV}{ }^{+} 05\right]$ enforcing information flow control, secBIP is a component-based modeling approach where non-interference is established at a more abstract level. Thus, secBIP can be apriori implemented using different programming languages and is independent from a specific operating system and execution platform.

Finally, it is worth mentioning that a lot of classical approaches fall short to handle information flow security [Zda04 for real systems. For $\sec B I P$ we privilege a very pragmatic approach and provide simple (syntactic) sufficient conditions allowing to automate the verification of non-interference. These conditions allow to eliminate a significant amount of security leakages, especially covert channels, independently from system language or the execution platform. However, these conditions can be very restrictive in some cases and a system designer may be interested to relax the non-interference properties.

\section{Conclusion and Future Work}

We present a MDS framework to secure component-based systems. We formally define two types of non-interference, respectively event and data noninterference. We provide a set of sufficient syntactic conditions which simplify verification of non-interference. These conditions are extensions of security typed language rules applied to our model. The use of our framework has been demonstrated to secure a web service application.

This work is currently being extended in two directions. First, we are investigating additional security conditions allowing to relax the non-interference property and control where downgrading can occur. Second, we are working towards the implementation of a complete design flow for secure systems based on secBIP. As a first step, we shall implement the verification method presented for annotated secBIP models. Then, use these models for generation of secure implementations, that is, executable code where the security properties are enforced by construction, at the generation time.

\section{References}

AL12. Accorsi, R., Lehmann, A.: Automatic information flow analysis of business process models. In: Barros, A., Gal, A., Kindler, E. (eds.) BPM 2012. LNCS, vol. 7481, pp. 172-187. Springer, Heidelberg (2012)

ASRL11. Abdellatif, T., Sfaxi, L., Robbana, R., Lakhnech, Y.: Automating information flow control in component-based distributed systems. In: 14th International ACM Sigsoft Symposium on Component Based Software Engineering (CBSE 2011), pp. 73-82. ACM (2011)

AWD11. Accorsi, R., Wonnemann, C., Dochow, S.: Swat: A security workflow analysis toolkit for reliably secure process-aware information systems. In: Sixth International Conference on Availability, Reliability and Security, ARES 2011, pp. 692-697. IEEE (2011) 
BBB ${ }^{+}$11. Basu, A., Bensalem, S., Bozga, M., Combaz, J., Jaber, M., Nguyen, T.-H., Sifakis, J.: Rigorous component-based design using the BIP framework. IEEE Software, Special Edition - Software Components beyond Programming - from Routines to Services 28(3), 41-48 (2011)

BBMP08. Bartolini, C., Bertolino, A., Marchetti, E., Parissis, I.: Data Flow-Based Validation of Web Services Compositions: Perspectives and Examples. In: de Lemos, R., Di Giandomenico, F., Gacek, C., Muccini, H., Vieira, M. (eds.) Architecting Dependable Systems V. LNCS, vol. 5135, pp. 298-325. Springer, Heidelberg (2008)

BBS06. Basu, A., Bozga, M., Sifakis, J.: Modeling Heterogeneous Real-time Systems in BIP. In: Fourth IEEE International Conference on Software Engineering and Formal Methods (SEFM 2006), pp. 3-12. IEEE Computer Society Press (2006)

BDL06. Basin, D., Doser, J., Lodderstedt, T.: Model driven security: from uml models to access control infrastructures. ACM Transactions on Software Engineering and Methodology 15, 39-91 (2006)

BLP76. Bell, E.D., La Padula, J.L.: Secure computer system: Unified exposition and Multics interpretation (1976)

DD77. Denning, D.E., Denning, P.J.: Certification of programs for secure information flow. Communications of the ACM, 504-513 (1977)

$\mathrm{EKV}^{+}$05. Efstathopoulos, P., Krohn, M., VanDeBogart, S., Frey, C., Ziegler, D., Kohler, E., Mazières, D., Kaashoek, F., Morris, R.: Labels and Event Processes in the Asbestos Operating System. SIGOPS Operating Systems Review 39(5), 17-30 (2005)

FG01. Focardi, R., Gorrieri, R.: Classification of Security Properties (Part I: Information Flow). In: Focardi, R., Gorrieri, R. (eds.) FOSAD 2000. LNCS, vol. 2171, pp. 331-396. Springer, Heidelberg (2001)

FGF09. Frau, S., Gorrieri, R., Ferigato, C.: Petri net security checker: Structural non-interference at work. In: Degano, P., Guttman, J., Martinelli, F. (eds.) FAST 2008. LNCS, vol. 5491, pp. 210-225. Springer, Heidelberg (2009)

FRS05. Focardi, R., Rossi, S., Sabelfeld, A.: Bridging language-based and process calculi security. In: Sassone, V. (ed.) FOSSACS 2005. LNCS, vol. 3441, pp. 299-315. Springer, Heidelberg (2005)

GM82. Goguen, J.A., Meseguer, J.: Security policies and security models. In: IEEE Symposium on Security and Privacy, pp. 11-20 (1982)

HV06. Hutter, D., Volkamer, M.: Information flow control to secure dynamic web service composition. In: Clark, J.A., Paige, R.F., Polack, F.A.C., Brooke, P.J. (eds.) SPC 2006. LNCS, vol. 3934, pp. 196-210. Springer, Heidelberg (2006)

jif. $\quad$ http://www.cs.cornell.edu/jif/

Kuh98. Richard Kuhn, D.: Role Based Access Control on MLS Systems without Kernel Changes. In: ACM Workshop on Role Based Access Control (RBAC 1998), pp. 25-32. ACM (1998)

$\mathrm{KYB}^{+}$07. Krohn, M., Yip, A., Brodsky, M., Cliffer, N., Frans Kaashoek, M., Kohler, E., Morris, R.: Information Flow Control for Standard OS Abstractions. SIGOPS Operating Systems Review 41(6), 321-334 (2007)

Man00. Mantel, H.: Possibilistic Definitions of Security - An Assembly Kit. In: 13th IEEE Workshop on Computer Security Foundations (CSFW 2000), p. 185. IEEE Computer Society (2000) 
McC88. McCullough, D.: Noninterference and the composability of security properties. In: Security and Privacy (SP 1988), pp. 177-186. IEEE Computer Society (1988)

McL94. McLean, J.: A general theory of composition for trace sets closed under selective interleaving functions. In: Security and Privacy (SP 1994), p. 79. IEEE Computer Society (1994)

Rus92. Rushby, J.: Noninterference, transitivity, and channel-control security policies. Technical Report CSL-92-2, SRI International (1992)

SM03. Sabelfeld, A., Myers, A.C.: Language-based information-flow security. IEEE Journal on Selected Areas in Communications 21(1) (2003)

SQSL05. Shen, J., Qing, S., Shen, Q., Li, L.: Covert channel identification founded on information flow analysis. In: Hao, Y., Liu, J., Wang, Y.-P., Cheung, Y.-M., Yin, H., Jiao, L., Ma, J., Jiao, Y.-C. (eds.) CIS 2005. LNCS (LNAI), vol. 3802, pp. 381-387. Springer, Heidelberg (2005)

SS01. Sabelfeld, A., Sands, D.: A per model of secure information flow in sequential programs. Higher Order Symbolic Computation 14(1), 59-91 (2001)

SS12. Seehusen, F., Stølen, K.: A Method for Model-driven Information Flow Security. In: Dependability and Computer Engineering: Concepts for Software-Intensive Systems, pp. 199-229. IGI Global (2012)

SSM98. Sandhu, R., Ravi, S., Munawer, Q.: How to do discretionary access control using roles. In: ACM Workshop on Role-Based Access Control (RBAC 1998), pp. 47-54. ACM (1998)

SV98. Smith, G., Volpano, D.: Secure information flow in a multi-threaded imperative language. In: Symposium on Principles of Programming Languages (POPL 1998), pp. 355-364. ACM (1998)

ZBWM08. Zeldovich, N., Boyd-Wickizer, S., Mazières, D.: Securing distributed systems with information flow control. In: 5th USENIX Symposium on Networked Systems Design and Implementation (NSDI 2008), pp. 293-308. USENIX Association (2008)

Zda04. Zdancewic, S.: Challenges for information-flow security. In: Programming Language Interference and Dependence, PLID 2004 (2004)

ZL97. Zakinthinos, A., Lee, E.S.: A general theory of security properties. In: Security and Privacy (SP 1997), pp. 94-102. IEEE Computer Society (1997)

ZZNM02. Zdancewic, S., Zheng, L., Nystrom, N., Myers, A.C.: Secure program partitioning. ACM Transactions on Computer Systems 20(3), 283-328 (2002)

\section{Appendix}

\section{Proof of Theorem 1}

Proof. We shall prove $\left.\operatorname{TRACES}\left(\gamma\left(B_{1}, \ldots, B_{n}\right), q_{0}\right)\right|_{s} ^{\sigma}=\operatorname{TRACES}\left(\left(\gamma \downarrow_{s}^{\sigma}\right)\left(B_{1}, \ldots\right.\right.$, $\left.\left.B_{n}\right), q_{0}\right)$ by double inclusion. " $\supseteq$ " inclusion: Independently of the unwinding relation, by using elementary set properties it holds that TRACES $\left(\left(\gamma \downarrow_{s}^{\sigma}\right)\left(B_{1}, \ldots\right.\right.$, $\left.\left.B_{n}\right), q_{0}\right)=\left.\left.\operatorname{TRACES}\left(\left(\gamma \downarrow_{s}^{\sigma}\right)\left(B_{1}, \ldots, B_{n}\right), q_{0}\right)\right|_{s} ^{\sigma} \subseteq \operatorname{TRACES}\left(\gamma\left(B_{1}, \ldots, B_{n}\right), q_{0}\right)\right|_{s} ^{\sigma}$. " $\subseteq$ " inclusion: This direction is an immediate consequence of the following Lemma 1. It states that for every trace $w$ in $\operatorname{TRACES}\left(\gamma\left(B_{1}, \ldots, B_{n}\right), q_{0}\right)$ its projection $\left.w\right|_{s} ^{\sigma}$ is also a valid trace in $\operatorname{TrACES}\left(\gamma\left(B_{1}, \ldots, B_{n}\right), q_{0}\right)$. But, this also means that $\left.w\right|_{s} ^{\sigma}$ is a valid trace in $\operatorname{TRACES}\left(\left(\gamma \downarrow_{s}^{\sigma}\right)\left(B_{1}, \ldots, B_{n}\right), q_{0}\right)$ which proves the result. 
Lemma 1. In the conditions of Theorem 1, for every trace $w$ in TRACES $\left(\gamma\left(B_{1}\right.\right.$, $\left.\left.\ldots, B_{n}\right), q_{0}\right)$, for every state $q$ such that $q_{0} \underset{C}{\stackrel{w}{\rightarrow}} q$, the projected trace $\left.w\right|_{s} ^{\sigma}$ is also a valid trace in $\operatorname{TRACES}\left(\gamma\left(B_{1}, \ldots, B_{n}\right), q_{0}\right)$ and moreover, for every state $q^{\prime}$ such that $q_{0} \underset{C}{\left.\stackrel{w}{\sigma}\right|_{s}} q^{\prime}$ it holds $q \sim_{s} q^{\prime}$.

Proof. The lemma is proved by induction on the length of the trace $w$. For the empty trace $w=\epsilon$ verification is trivial: $\sim_{s}$ holds for the initial state $q_{0} \sim_{s} q_{0}$ and $\epsilon=\left.\epsilon\right|_{s} ^{\sigma}$. By induction hypothesis, let assume the property holds for traces of length $n$. We shall prove the property for traces of length $n+1$. Let $w^{\prime}=w a$ be an arbitrary trace of length $n+1$, let $w$ be its prefix (trace) of length $n$ and let $a$ be the last interaction. Consider states $q, q_{1}$ such that $q_{0} \underset{C}{\stackrel{w}{\longrightarrow}} q \underset{C}{\stackrel{a}{\longrightarrow}} q_{1}$. By the induction hypothesis we know that $\left.w\right|_{s} ^{\sigma}$ is a valid trace and for all states $q^{\prime}$ such that $q_{0} \underset{C}{\stackrel{\left.w\right|_{s} ^{\sigma}}{\longrightarrow}} q^{\prime}$ it holds $q \sim_{s} q^{\prime}$. We distinguish two cases, depending on the security level of $a$ :
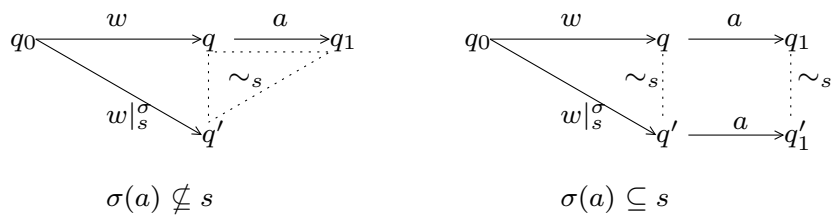

Fig. 8. Proof illustration for Lemma 1

- $\sigma(a) \nsubseteq s$ : In this case, $\left.w^{\prime}\right|_{s} ^{\sigma}=\left.w\right|_{s} ^{\sigma}$ hence, $\left.w^{\prime}\right|_{s} ^{\sigma}$ is a valid trace as well, reaching the same states $q^{\prime}$. Moreover, since $a$ is invisible for $s$, the unwinding condition (1) ensures that $q \sim_{s} q_{1}$. By transitivity, this implies that $q_{1} \sim_{s} q^{\prime}$, which proves the result.

- $\sigma(a) \subseteq s$ : In this case, $\left.w^{\prime}\right|_{s} ^{\sigma}=\left.w\right|_{s} ^{\sigma} a$. From the unwinding condition (2), since $q \sim_{s} q^{\prime}$ and $a$ is visible and enabled in $q$ then, $a$ must also be enabled in $q^{\prime}$. Therefore, $\left.w\right|_{s} ^{\sigma}$ can be extended with $a$ from state $q^{\prime}$ to some $q_{1}^{\prime}$ hence, $\left.w^{\prime}\right|_{s} ^{\sigma}$ is indeed a valid trace. Moreover, since $q \sim_{s} q^{\prime}$ the unwinding condition (2) ensures also that $q_{1} \sim_{s} q_{1}^{\prime}$, which proves the result.

\section{Proof of Theorem 2}

Proof. Let us consider two equivalent states $q_{1} \approx_{s}^{\sigma} q_{2}$.

The first condition for data non-interference requires that, for any trace $w_{1}$ from $q_{1}$ there exists a trace $w_{2}$ from $q_{2}$ having the same projection at level $s$, that is, $\left.w_{1}\right|_{s} ^{\sigma}=\left.w_{2}\right|_{s} ^{\sigma}$.

We shall prove a slightly stronger property, namely, the trace $w_{2}$ can be chosen such that, the successors $q_{1}^{\prime}$ and $q_{2}^{\prime}$ of respectively $q_{1}$ by $w_{1}$ and $q_{2}$ by $w_{2}$ are moreover equivalent, that is, $q_{1}^{\prime} \approx_{s}^{\sigma} q_{2}^{\prime}$. The proof is by induction on the length of 
the trace $w_{1}$. The base case: for the empty trace $w_{1}=\epsilon$ we take equally $w_{2}=\epsilon$ we immediately have $q_{1}^{\prime}=q_{1} \approx_{s}^{\sigma} q_{2}=q_{2}^{\prime}$. The induction step: we assume, by induction hypothesis that the property holds for all traces $w_{1}$ such that $\left|w_{1}\right| \leq n$ and we shall prove it for all traces $w_{1}^{\prime}$ such that $\left|w_{1}^{\prime}\right|=n+1$. Let $a$ be the last interaction executed in $w_{1}^{\prime}$, that is, $w_{1}^{\prime}=w_{1} a$ with $\left|w_{1}\right|=n$. Let $q_{1}^{\prime \prime}$ be the state reached from $q_{1}$ by $w_{1}$. From the induction hypothesis, there exists a trace $w_{2}$ that leads $q_{2}$ into $q_{2}^{\prime \prime}$ such that $\left.w_{1}\right|_{s} ^{\sigma}=\left.w_{2}\right|_{s} ^{\sigma}$ and moreover $q_{1}^{\prime \prime} \approx_{s}^{\sigma} q_{2}^{\prime \prime}$. We distinguish two cases, depending on the security level of $a$ :

- $\sigma(a) \not \subset s$ : since $\approx_{s}^{\sigma}$ is unwinding and $q_{1}^{\prime \prime} \underset{C}{\vec{a}} q_{1}^{\prime}$ it follows that $q_{1}^{\prime \prime} \approx_{s}^{\sigma} q_{1}^{\prime}$. In this case, we take $w_{2}^{\prime}=w_{2}$ and $q_{2}^{\prime}=q_{2}^{\prime \prime}$ which ensures both $\left.w_{1}^{\prime}\right|_{s} ^{\sigma}=\left.w_{1}\right|_{s} ^{\sigma}=$ $\left.w_{2}\right|_{s} ^{\sigma}=\left.w_{2}^{\prime}\right|_{s} ^{\sigma}$ and $q_{1}^{\prime} \approx_{s}^{\sigma} q_{1}^{\prime \prime} \approx q_{2}^{\prime \prime}=q_{2}^{\prime}$.

$-\sigma(a) \subseteq s:$ since $\approx_{s}^{\sigma}$ is unwinding and $q_{1}^{\prime \prime} \approx_{s}^{\sigma} q_{2}^{\prime \prime}$ and $q_{1}^{\prime \prime} \underset{C}{\vec{C}} q_{1}^{\prime}$ there must exists $q_{2}^{\prime}$ such that $q_{2}^{\prime \prime} \underset{C}{\stackrel{a}{\longrightarrow}} q_{2}^{\prime}$ and moreover, for any such choice $q_{1}^{\prime} \approx_{s}^{\sigma} q_{2}^{\prime}$. Hence, in this case, the trace $w_{2}^{\prime}=w_{2} a$ executed from $q_{2}$ and leading to $q_{2}^{\prime}$ satisfies our property, namely $\left.w_{1}^{\prime}\right|_{s} ^{\sigma}=\left.w_{1}\right|_{s} ^{\sigma} a=\left.w_{2}\right|_{s} ^{\sigma} a=\left.w_{2}^{\prime}\right|_{s} ^{\sigma}$ and $q_{1}^{\prime} \approx_{s}^{\sigma} q_{2}^{\prime}$.

The second condition for data non-interference requires that, for any traces $w_{1}$ and $w_{2}$ with equal projection on security level $s$, that is $\left.w_{1}\right|_{s} ^{\sigma}=\left.w_{2}\right|_{s} ^{\sigma}$, any successor states $q_{1}^{\prime}$ and $q_{2}^{\prime}$ of respectively $q_{1}$ by $w_{1}$ and $q_{2}$ by $w_{2}$ are also equivalent at level $s$. This property is proved also by induction on $\left|w_{1}\right|+\left|w_{2}\right|$, that is, on the sum of the lengths of traces $w_{1}, w_{2}$. The base case: for empty traces $w_{1}=w_{2}=\epsilon$ we have that $q_{1}^{\prime}=q_{1}$ and $q_{2}^{\prime}=q_{2}$ and hence trivially $q_{1}^{\prime} \approx_{s}^{\sigma} q_{2}^{\prime}$. The induction step: we assume, by induction hypothesis that the property holds for any traces $w_{1}, w_{2}$ such that $\left|w_{1}\right|+\left|w_{2}\right| \leq n$ and we shall prove it for all traces $w_{1}^{\prime}, w_{2}^{\prime}$ such that $\left|w_{1}^{\prime}\right|+\left|w_{2}^{\prime}\right|=n+1$. We distinguish two cases, depending on the security levels of the last interactions occurring in $w_{1}^{\prime}$ and $w_{2}^{\prime}$.
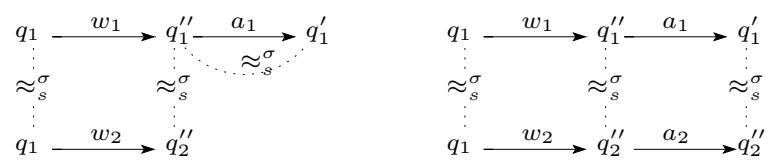

Fig. 9. Proof illustration for Theorem 2

- at least one of the last interactions in $w_{1}^{\prime}$ or $w_{2}^{\prime}$ has a security level not lower or equal to $s$. W.l.o.g, consider that indeed $w_{1}^{\prime}=w_{1} a_{1}$ and $\sigma\left(a_{1}\right) \nsubseteq s$. This situation is depicted in Figure 9] (left).

Let $q_{1}^{\prime \prime}$ be the state reached from $q_{1}$ after $w_{1}$. Since $\left.w_{1}^{\prime}\right|_{s} ^{\sigma}=\left.w_{2}^{\prime}\right|_{s} ^{\sigma}$ and $\sigma\left(a_{1}\right) \nsubseteq s$ it follows that $\left.w_{1}\right|_{s} ^{\sigma}=\left.w_{1}^{\prime}\right|_{s} ^{\sigma}=\left.w_{2}^{\prime}\right|_{s} ^{\sigma}$. The induction hypothesis holds then for $w_{1}$ and $w_{2}^{\prime}$ because $\left|w_{1}\right|+\left|w_{2}^{\prime}\right|=n-1$ and hence we have that $q_{1}^{\prime \prime} \approx_{s}^{\sigma} q_{2}^{\prime}$. Moreover, $q_{1}^{\prime}$ is a successor of $q_{1}^{\prime \prime}$ by interaction $a_{1}$. Since the security level of $a_{1}$ is not lower or equal to $s$, and $\approx_{s}^{\sigma}$ is an unwinding relation at level $s$, it follows from the local consistency condition that $q_{1}^{\prime \prime} \approx_{s}^{\sigma} q_{1}$. Then, by transitivity of $\approx_{s}^{\sigma}$ we obtain that $q_{1}^{\prime} \approx_{s}^{\sigma} q_{2}^{\prime}$. 
- the last interactions of both traces $w_{1}^{\prime}$ and $w_{2}^{\prime}$ have security level lower or equal to $s$. That is, consider $w_{1}^{\prime}=w_{1} a_{1}$ and $w_{2}^{\prime}=w_{2} a_{2}$ with $\sigma\left(a_{1}\right) \subseteq s$, $\sigma\left(a_{2}\right) \subseteq s$. This situation is depicted in Figure 9. (right).

Let $q_{1}^{\prime \prime}$ and $q_{2}^{\prime \prime}$ be the states reached respectively from $q_{1}$ by $w_{1}$ and from $q_{2}$ by $w_{2}$. Since $\sigma\left(a_{1}\right) \subseteq s, \sigma\left(a_{2}\right) \subseteq s$ we have $\left.w_{1}^{\prime}\right|_{s} ^{\sigma}=\left.w_{1}\right|_{s} ^{\sigma} a_{1},\left.w_{2}^{\prime}\right|_{s} ^{\sigma}=\left.w_{2}\right|_{s} ^{\sigma} a_{2}$. From the hypothesis, $\left.w_{1}^{\prime}\right|_{s} ^{\sigma}=\left.w_{2}^{\prime}\right|_{s} ^{\sigma}$, it follows that both $a_{1}=a_{2}$ and $\left.w_{1}\right|_{s} ^{\sigma}=$ $\left.w_{2}\right|_{s} ^{\sigma}$. Therefore, the induction hypothesis can be applied for traces $w_{1}, w_{2}$ because $\left|w_{1}\right|+\left|w_{2}\right|=n-2$ and hence, we obtain $q_{1}^{\prime \prime} \approx_{s}^{\sigma} q_{2}^{\prime \prime}$. But now, $q_{1}^{\prime}$ and $q_{2}^{\prime}$ are immediate successors of two equivalent states $q_{1}^{\prime \prime}$ and $q_{2}^{\prime \prime}$ by executing some interaction $a=a_{1}=a_{2}$, having security level lower or equal to $s$. Since, $\approx_{s}^{\sigma}$ is an unwinding relation at level $s$, it follows from the step consistency condition that successors states $q_{1}^{\prime}$ and $q_{2}^{\prime}$ are also equivalent at level $s$, hence, $q_{1}^{\prime} \approx_{s}^{\sigma} q_{2}^{\prime}$.

\section{Proof of Theorem 3}

Proof. Let $s$ be an arbitrary fixed security level. We shall prove that $\approx_{s}^{\sigma}$ satisfies the local, output and step consistency, as required by Definition [11.

local consistency: Let $q, q^{\prime} \in Q_{C}$ be two states such that $q \underset{C}{\stackrel{a}{C}} q^{\prime}$. We must show that if $\sigma(a) \nsubseteq s$ then $q \approx_{s}^{\sigma} q^{\prime}$.

All variables $x$ modified by $a$ itself and by the transitions participating in $a$ are such that $\sigma(a) \subseteq \sigma(x)$ (security conditions, $(i i i)$ ). Then, since $\sigma(a) \nsubseteq s$, it also follows that all variables modified have security level greater or incomparable to $s$. Conversely, it follows that all variables with security levels lower or equal to $s$ are not modified by $a$, hence they have the same values in $q$ in $q^{\prime}$.

Regarding control locations, we proceed by contradiction. Let consider that some component $B_{i}$ is respectively at $\ell_{i}$ in $q$ and at $\ell_{i}^{\prime}$ in $q^{\prime}$ and moreover, either at $\ell_{i}$ or $\ell_{i}^{\prime}$ there exists transitions with ports having security levels lower or equal $s$. Since the location of $B_{i}$ has changed, it means that it has participated in the interaction $a$ using some transition $\tau_{i}=\ell_{i} \stackrel{p_{i}}{\longrightarrow} \ell_{i}^{\prime}$. Let consider the two situations:

- there exists transitions with security level lower or equal to $s$ at $\ell_{i}^{\prime}$. Let $\tau_{i}^{\prime}=\ell_{i}^{\prime} \stackrel{p_{i}^{\prime}}{\longrightarrow} \ell_{i}^{\prime \prime}$ such a transition. This situation contradicts the security conditions $(i)$, as $\tau_{i}^{\prime}$ is causally dependent on $\tau_{i}$ and has a different, yet not increased security level i.e., $\sigma\left(p_{i}\right)=\sigma(a) \nsubseteq s$ and $\sigma\left(p_{i}^{\prime}\right) \subseteq s$.

- there exists transitions with security level lower or equal to $s$ at $\ell_{i}$. Let $\tau_{i}^{\prime}=\ell_{i} \stackrel{p_{i}^{\prime}}{\longrightarrow} \ell_{i}^{\prime \prime}$ such a transition. This situation contradicts again the security conditions $(i)$ : as $\tau_{i}$ and $\tau_{i}^{\prime}$ are now conflicting it must be $\sigma\left(p_{i}\right) \subseteq \sigma\left(p_{i}^{\prime}\right)$ which contradicts that $\sigma\left(p_{i}\right)=\sigma(a) \nsubseteq s$ and $\sigma\left(p_{i}^{\prime}\right) \subseteq s$.

Henceforth, as the two situations lead to contradiction we conclude that, either $\ell_{i}=\ell_{i}^{\prime}$, or otherwise, neither in $\ell_{i}$ or $\ell_{i}^{\prime}$ there exists transitions with ports having security level lower or equal to $s$. This conclude the proof of $q \approx_{s}^{\sigma} q^{\prime}$

output and step consistency: Let $q_{1}, q_{2}$ be two equivalent states $q_{1} \approx_{s}^{\sigma} q_{2}$.

Let $a$ be an interaction with security level lower or equal to $s$ enabled in $q_{1}$. We show that the same interaction is enabled in $q_{2}$. All components participating in 
$a$ use transitions with ports with the same level as $a$, hence at most $s$. Therefore, these components are at control locations where there are outgoing transitions with level at most $s$. Then, these components are precisely in the same locations in $q_{2}$ since $q_{1} \approx_{s}^{\sigma} q_{2}$.

Moreover, all the guards of the interacting transitions as well as the guard of the interaction use variables with security level lower or equal to $\sigma(a)$ and consequently, lower or equal to $s$ (security conditions, (iii)). But again, $q_{1} \approx_{s}^{\sigma} q_{2}$ implies that all variables with levels lower or equal to $s$ have equal values in $q_{1}$ and $q_{2}$. Hence, the guards used in $a$ have the same evaluation in $q_{1}$ or $q_{2}$. Together with equality on control locations, established earlier, this implies that $a$ is enabled in $q_{2}$.

Let now consider two arbitrary states $q_{1}^{\prime}, q_{2}^{\prime}$ reached by a from respectively $q_{1}$ and $q_{2}$. We must show that $q_{1}^{\prime} \approx_{s}^{\sigma} q_{2}^{\prime}$. First, as $\sigma(a) \subseteq s$, it follows that, as explained before, enabledness of $a$ depends exclusively on identical parts of $q_{1}$ and $q_{2}$. Moreover, due to security conditions $(i v)$ it follows also that the execution of $a$ synchronizes exactly the same set of transitions when executed either from $q_{1}$ or from $q_{2}$. Hence, in the successor states $q_{1}^{\prime}$ and $q_{2}^{\prime}$ all interacting atomic components have moved towards the same locations. The equality condition on the control locations is therefore satisfied. Furthermore, using security conditions (ii) it holds that all variables modified by transitions involved in $a$, if they have security values lower or equal to $s$, they will be assigned the same values. That is, the assigned expression use only variables with a lower security level, and hence identical on $q_{1}$ and $q_{2}$. This ensures equality of variables with security level lower or equal to $s$ in $q_{1}^{\prime}$ and $q_{2}^{\prime}$, which conclude the proof. 